\title{
Near-Extremal Black Hole Evaporation in Asymptotically Flat Spacetime
}

\author{
Kamran Diba* and David A. Lowe \\ Department of Physics, \\ Brown University, \\ Providence, RI 02912, \\ U.S.A.
}

\begin{abstract}
We study black hole evaporation of near-extremal black holes in spherically reduced models with asymptotically Minkowskian spacetime, with the effects of the back-reaction on the geometry included semi-classically. The stress-energy tensor is calculated for null in-falling observers. It is shown that the evaporation proceeds smoothly and there are no instabilities of the outer or inner apparent horizon before the endpoint of evaporation.
\end{abstract}

\section{INTRODUCTION}

The evolution of near-extremal black holes has important consequences for the resolution of the information paradox. Extremal black holes are potential candidates for endpoint remnants [1]2], and can potentially store information behind the double horizon [3]. In addition, study of their evaporation avoids the naked singularities observed in models of linear dilaton black hole evaporation [4,5], which are likely typical of the evolution of uncharged black holes..

However, it has been suggested by Jacobson, using adiabatic arguments, that the semi-classical evolution of nearextremal black holes may break down while still far from extremality [6]. The claim is that in-falling photons created at the outer apparent horizon during Hawking evaporation will eventually fall through the inner horizon and either encounter a large buildup of energy behind the inner horizon, or alternatively, pile up behind the outer horizon, causing it to become unstable. Thus, in either scenario, the semiclassical approximation may break down long before one would expect based on thermodynamic/statistical mechanics arguments [7].

This claim has been explored in an analytical model [8] using a near-horizon Reissner-Norsdröm model originally proposed in [9.10], and further elaborated in [11,12]. No instabilities were observed in that case. However, the boundary conditions there were computationally motivated rather than physically motivated. In particular there was no obvious extension to asymptotically flat spacetime.

In the present work we numerically explore semi-classical evaporation in two spherically-reduced two-dimensional models of asymptotically Minkowskian near-extremal black holes. We find results consistent with [8], indicating that semi-classically there are no horizon instabilities during the evaporation of charged black holes.

\section{THE RN MODEL}

For our first model [13 15], we begin with the Einstein-Hilbert action

$$
S=\frac{1}{2 \pi} \int d^{2} x \sqrt{-g} e^{-2 \phi}\left[R+2(\nabla \phi)^{2}+2 \lambda^{2} e^{2 \phi}\right] .
$$

To this, we add an electromagnetic contribution:

$$
S_{E M}=-\frac{1}{2 \pi} \int d^{2} x \sqrt{-g} Q^{2} e^{2 \phi},
$$

where $Q$ is the charge of the black hole as it appears in the spherically symmetric Reissner-Nordström metric,

$$
d s^{2}=-\left(1-\frac{2 M}{r}+\frac{Q^{2}}{r^{2}}\right) d t^{2}+\frac{1}{1-\frac{2 M}{r}+\frac{Q^{2}}{r^{2}}} d r^{2} .
$$

The effective two dimensional action that describes (3) is given by

*e-mail: diba@het.brown.edu 


$$
S=\frac{1}{2 \pi} \int d^{2} x \sqrt{-g} e^{-2 \phi}\left[R+2(\nabla \phi)^{2}+2 \lambda^{2} e^{2 \phi}-Q^{2} e^{4 \phi}\right]
$$

In order to study the dynamics, we add a large number $N$ of matter fields, $f_{i}$,

$$
S_{M}=-\frac{1}{4 \pi} \sum_{i=1}^{N} \int d^{2} x \sqrt{-g}\left(\nabla f_{i}\right)^{2} .
$$

This form of coupling is mostly justified by the ease of the resulting solutions. However, it also corresponds to the bosonized version of the Callan-Rubakov modes of fermions coupled to a magnetically charged black in four dimensions [16].

Additionally, we include the Liouville-Polyakov term

$$
S_{Q}=-\frac{N \hbar}{96 \pi} \int d^{2} x \sqrt{-g} R \square^{-1} R,
$$

which effectively accounts for the back-reaction of the matter stress-energy tensor on the space-time geometry [19,20].

The full lagrangian in the conformal gauge becomes

$$
\mathcal{L}=e^{-2 \phi}\left(4 \partial_{+} \partial_{-} \rho-4 \partial_{+} \phi \partial_{-} \phi\right)+\lambda^{2} e^{2 \rho}-Q^{2} e^{2 \rho+2 \phi}-2 \kappa \partial_{+} \rho \partial_{-} \rho .
$$

¿From this point on, we choose $\lambda=1$.

\section{A. Equations of Motion}

Variation of (7) with respect to $\phi$ gives

$$
\partial_{+} \partial_{-} \rho-\partial_{+} \partial_{-} \phi+\partial_{+} \phi \partial_{-} \phi+\frac{Q^{2}}{4} e^{2 \rho+4 \phi}=0 .
$$

A $\rho$ variation gives

$$
\frac{\kappa}{2} e^{2 \phi} \partial_{+} \partial_{-} \rho-\partial_{+} \partial_{-} \phi+2 \partial_{+} \phi \partial_{-} \phi+\frac{e^{2 \rho}}{4}\left(e^{2 \phi}-Q^{2} e^{4 \phi}\right) .
$$

The resultant equations of motion can be written as

$$
\begin{aligned}
& \partial_{+} \partial_{-} \rho=\frac{\partial_{+} \phi \partial_{-} \phi+\frac{1}{4} e^{2 \rho}\left(e^{2 \phi}-2 Q^{2} e^{4 \phi}\right)}{1-\frac{\kappa}{2} e^{2 \phi}} \\
& \partial_{+} \partial_{-} \phi=\partial_{+} \partial_{-} \rho+\partial_{+} \phi \partial_{-} \phi+\frac{Q^{2}}{4} e^{2 \rho+4 \phi}
\end{aligned}
$$

We may note that this model possesses a singularity for $e^{-2 \phi}=\kappa / 2$. In our solutions, this singularity will lie safely behind the inner horizon. It is useful to rewrite these equations with $\tilde{\phi}=e^{-\phi}$. Thus $\partial_{+} \tilde{\phi}=-\partial_{+} \phi \tilde{\phi}$ and $\partial_{-} \partial_{+} \tilde{\phi}=-\partial_{-} \partial_{+} \phi \tilde{\phi}+\partial_{-} \phi \partial_{+} \phi \tilde{\phi}$. We can rewrite (11),

$$
\partial_{+} \partial_{-} \phi-\partial_{+} \phi \partial_{-} \phi=\frac{Q^{2}}{4} e^{2 \rho+4 \phi}+\frac{\partial_{+} \phi \partial_{-} \phi+\frac{e^{2 \rho}}{4}\left(e^{2 \phi}-2 Q^{2} e^{4 \phi}\right)}{1-\frac{\kappa}{2} e^{2 \phi}}
$$

as

$$
-\frac{\partial_{+} \partial_{-} \tilde{\phi}}{\tilde{\phi}}=\frac{Q^{2}}{4} \frac{e^{2 \rho}}{\tilde{\phi}^{4}}+\left(\frac{\partial_{+} \tilde{\phi} \partial_{-} \tilde{\phi}}{\tilde{\phi}^{2}}+\frac{e^{2 \rho}}{4 \tilde{\phi}^{2}}-\frac{Q^{2} e^{2 \rho}}{2 \tilde{\phi}^{4}}\right) /\left(1-\frac{\kappa}{2 \tilde{\phi}^{2}}\right) .
$$

Hence

$$
\partial_{+} \partial_{-} \tilde{\phi}=-\frac{Q^{2}}{4} \frac{e^{2 \rho}}{\tilde{\phi}^{3}}-\frac{\tilde{\phi} \partial_{+} \tilde{\phi} \partial_{-} \tilde{\phi}+\frac{e^{2 \rho} \tilde{\phi}}{4}-\frac{Q^{2} e^{2 \rho}}{2 \tilde{\phi}}}{\tilde{\phi}^{2}-\kappa / 2}
$$


and,

$$
\begin{aligned}
\partial_{+} \partial_{-} \rho & =\partial_{+} \partial_{-} \phi-\partial_{+} \phi \partial_{-} \phi-\frac{Q^{2}}{4} e^{2 \rho+4 \phi} \\
& =-\frac{\partial_{+} \partial_{-} \tilde{\phi}}{\tilde{\phi}}-\frac{Q^{2} e^{2 \rho}}{4 \tilde{\phi}^{4}} .
\end{aligned}
$$

More concisely,

$$
\begin{aligned}
\partial_{+} \partial_{-} \rho & =\frac{\partial_{+} \tilde{\phi} \partial_{-} \tilde{\phi}+\frac{e^{2} \rho}{4}\left(1-\frac{2 Q^{2}}{\tilde{\phi}^{2}}\right)}{\tilde{\phi}^{2}-\kappa / 2}, \\
\partial_{+} \partial_{-} \tilde{\phi} & =-\left(\partial_{+} \partial_{-} \rho\right) \tilde{\phi}-\frac{Q^{2} e^{2 \rho}}{4 \tilde{\phi}^{3}} .
\end{aligned}
$$

\section{B. The Static Solution}

We first solve the equations of motion in the limit of the static extremal black hole [13]. (See also [17] for a recent discussion of these static solutions, and [18] for analysis of the full four-dimensional semi-classical equations of motion, in the near-horizon limit.) We will later need these as boundary conditions for the dynamic solutions. The model possesses a linear dilaton vacuum solution corresponding to

$$
\begin{aligned}
& \phi=-\log \frac{1}{2}\left(x^{+}-x^{-}\right), \\
& \rho=0 .
\end{aligned}
$$

This suggests to us a reasonable choice for the radial coordinate:

$$
\sigma=\frac{x^{+}-x^{-}}{2} .
$$

We note that

$$
\frac{\partial \sigma}{\partial x^{+}}=-\frac{\partial \sigma}{\partial x^{-}}=\frac{1}{2}
$$

The equations of motion, $(17 \sqrt{18})$, become

$$
\begin{aligned}
& \rho^{\prime \prime}=\frac{\left(\tilde{\phi}^{\prime}\right)^{2}-e^{2 \rho}\left(1-\frac{2 Q^{2}}{\tilde{\phi}^{2}}\right)}{\tilde{\phi}^{2}-\kappa / 2}, \\
& \tilde{\phi}^{\prime \prime}=-\rho^{\prime \prime} \tilde{\phi}+\frac{Q^{2} e^{2 \rho}}{\tilde{\phi}^{3}}
\end{aligned}
$$

with the constraint that

$$
2 \tilde{\phi}\left(\tilde{\phi}^{\prime \prime}-2 \rho^{\prime} \tilde{\phi}^{\prime \prime}\right)+\kappa\left(\rho^{\prime \prime}-\left(\rho^{\prime}\right)^{2}+t\right)=0 .
$$

Linearizing about (19), as $\sigma \rightarrow \infty$ we see that

$$
\begin{aligned}
\sigma^{2} \delta \rho^{\prime \prime} & =1+2 \delta \tilde{\phi}^{\prime}-(1+2 \delta \rho) \\
& =2 \delta \tilde{\phi}^{\prime}+2 \delta \rho,
\end{aligned}
$$

and

$$
\delta \tilde{\phi}^{\prime \prime}=-\sigma \delta \rho^{\prime \prime}
$$

We can see that 


$$
\delta \tilde{\phi}^{\prime \prime}=2 \delta \rho^{\prime}=-\sigma \delta \rho^{\prime \prime}
$$

has the solution

$$
\begin{aligned}
\delta \rho^{\prime} & =\frac{M}{\sigma^{2}}, \\
\delta \tilde{\phi}^{\prime} & =1-\frac{2 M}{\sigma} .
\end{aligned}
$$

As $\sigma \rightarrow \infty$ the linearized vacuum solutions are therefore

$$
\begin{aligned}
& \rho=-\frac{M}{\sigma}, \\
& \tilde{\phi}=\sigma-2 M \log \sigma .
\end{aligned}
$$

\section{The Boundary Conditions}

We will study black holes perturbed by shock waves of the matter fields $f_{i}$. Our boundary conditions are chosen such that to the past of $x^{+}=0$, we have the static extremal solution of (23), (24) with the initial conditions at a chosen large value of $\sigma=\sigma_{\infty}$ determined by (32), (33). In practice, we make choices for $Q$ and $\kappa$, and adjust $M$

so that a double horizon appears in the interior region of the solutions. Along a large value of $x^{-}=x_{\infty}^{-}$, and to the future of $x^{+}=0$, we then impose the asymptotic linearized solutions of a black hole with mass $M+\Delta M$

$$
\begin{gathered}
\partial_{+} \rho=2 \frac{M+\Delta M}{\left(x^{+}-x^{-}\right)^{2}}, \\
\partial_{+} \tilde{\phi}=\frac{1}{2}-2 \frac{M+\Delta M}{x^{-}-x^{-}} .
\end{gathered}
$$

\section{THE DW MODEL}

The second model we consider, the so-called DW model, derives from a general class of two-dimensional renormalizable generally covariant field theories [13,21]

$$
\mathcal{L}_{c l}=\sqrt{-g}\left(D(\phi) R+G(\phi)(\nabla \phi)^{2}+H(\phi)\right) .
$$

We require that the potentials in (36) behave asymptotically like those of the linear dilaton gravity model considered above. That is,

$$
D(\phi) \longrightarrow \frac{G(\phi)}{4} \longrightarrow \frac{H(\phi)}{4} \longrightarrow e^{-2 \phi}
$$

as $\phi \rightarrow-\infty$. After requiring, without loss of generality, that $G(\phi)=-2 D^{\prime}(\phi)$ and performing a Brans-Dicke transformation on the metric $\hat{g}=e^{-2 \phi} g$, the Lagrangian can be rewritten as

$$
\hat{\mathcal{L}}_{c l}=\sqrt{-\hat{g}}(D(\phi) \hat{R}+W(\phi)),
$$

where $W(\phi)=e^{2 \phi} H(\phi)$. This is the form of the lagrangian we refer to as the DW model. For these solutions, it is convenient to work in the conformal gauge,

$$
d s^{2}=-e^{2 \rho} d x^{+} d x^{-} .
$$

Again, we add $N$ matter fields, (5), and a back-reaction term, (5). Now

$$
\begin{aligned}
\mathcal{L} & =\mathcal{L}_{c l}+\sum_{i=1}^{N} \partial_{+} f_{i} \partial_{-} f_{i}-2 \kappa \partial_{+} \rho \partial_{-} \rho \\
& =4 D \partial_{+} \partial_{-} \rho+4 D^{\prime} \partial_{+} \phi \partial_{-} \phi+\frac{W}{2} e^{2 \rho-2 \phi}-2 \kappa \partial_{+} \rho \partial_{-} \rho+\sum_{i=1}^{N} \partial_{+} f_{i} \partial_{-} f_{i}
\end{aligned}
$$

where we have let $\kappa=N \hbar / 12$. 


\section{A. Equations of Motion}

Variation of (40) with respect to $\rho$ and $\phi$ yields

$$
4 D^{\prime \prime} \partial_{+} \phi \partial_{-} \phi+4 D^{\prime} \partial_{+} \partial_{-} \phi+W e^{2 \rho-2 \phi}+4 \kappa \partial_{+} \partial_{-} \rho=0,
$$

and

$$
4 D^{\prime} \partial_{+} \partial_{-} \rho+4 D^{\prime \prime} \partial_{+} \phi \partial_{-} \phi-8 D^{\prime} \partial_{+} \partial_{-} \phi-8 D^{\prime \prime} \partial_{+} \phi \partial_{-} \phi-\left(W-W^{\prime} / 2\right) e^{2 \rho-2 \phi}=0
$$

respectively. Reexpressing these in a more useful form, we obtain the equations of motion:

$$
\begin{aligned}
& \partial_{+} \partial_{-} \phi=-\frac{D^{\prime \prime} \partial_{+} \phi \partial_{-} \phi+\frac{1}{4} e^{2 \rho-2 \phi} W+\kappa \partial_{+} \partial_{-} \rho}{D^{\prime}}, \\
& \partial_{+} \partial_{-} \rho=\frac{-D^{\prime \prime} \partial_{+} \phi \partial_{-} \phi-\frac{1}{4} e^{2 \rho-2 \phi}\left(W+\frac{W^{\prime}}{2}\right)}{D^{\prime}+2 \kappa} .
\end{aligned}
$$

As discussed in [21], we must choose $D$ such that $D^{\prime}(\phi)<0$ for all $\phi$ in order to avoid a singularity of the Brans-Dicke transformation. Also,

$$
\frac{d \phi}{d \sigma} \sim \int d \phi W(\phi) D^{\prime}(\phi)
$$

has zeroes along $\sigma$, the radial coordinate, equal to the number of zeroes of $W(\phi) D^{\prime}(\phi)$ plus one. Therefore, for a charged black hole, $W(\phi) D^{\prime}(\phi)$ must possess a single zero, for which $\phi(\sigma)$ will correspond to the extremal radius. In our solutions, we investigate the choice where $D=e^{-2 \phi}-\gamma^{2} e^{2 \phi}$ and $W=4-\mu^{2} e^{4 \phi} \cdot \mu^{2}$, as the coefficient of $e^{2 \phi}$, behaves as the charge of the black hole. This becomes clear through comparison with (2), from the Reissner-Nordström model.

\section{B. The Boundary Conditions}

The boundary conditions are set so that to the past of $x_{0}^{+}=1$ the solution corresponds to the static, extremal solution of the equations of motion, while along $x^{-}=-\infty$, and to the future of $x_{0}^{+}=1$, the boundary conditions are

$$
\begin{aligned}
\phi=\rho & =-\frac{1}{2} \log \left(M+\Delta M\left(x_{0}^{+}-x^{+}\right)-x^{+} x^{-}\right), \\
\partial_{+} \phi=\partial_{+} \rho & =\frac{x^{-}+\Delta M}{2\left(M+\Delta M x_{0}^{+}-x^{+} x^{-}-\Delta M x^{+}\right)} .
\end{aligned}
$$

This is the same boundary condition imposed in [4]. In practice, the static solutions are solved with the static form of (44) and (45), imposed at some large value of $x^{-}=x_{\infty}^{-}$. Then, $\mathrm{M}$ is fine-tuned so that the there is a double horizon static solution to the equations of motion, (44) and (45). This solution in turn becomes the boundary condition at $x_{0}^{+}=1$ for the full dynamical solutions.

\section{AFFINE COORDINATES}

We would like to answer questions regarding black hole stability during the evaporation process. An appropriate indicator would be the behavior of the stress-energy as observed along a null affinely parameterized geodesic. The Christoffel symbols for the metric (39) are

$$
\begin{aligned}
& \Gamma_{++}^{+}=2 \partial_{+} \rho, \\
& \Gamma_{--}^{-}=2 \partial_{-} \rho .
\end{aligned}
$$

¿From the geodesic equation for $x^{+}$, we obtain

$$
\frac{d^{2} x^{+}}{d \tilde{x}+^{2}}+2 \partial_{+} \rho \frac{d x^{+}}{d \tilde{x}^{+}} \frac{d x^{+}}{d \tilde{x}^{+}}=0
$$


with a similar equation for $x^{-} . \tilde{x}^{+}$is an affinely parameterized null geodesic. If $x^{-}$is held fixed at $x^{-}=x_{0}^{-}$,

$$
\partial_{+} \rho \frac{d x^{+}}{d \tilde{x}^{+}}=\frac{d \rho}{d \tilde{x}^{+}} .
$$

Then (51) reduces to

$$
\frac{d^{2} x^{+}}{d \tilde{x}^{+2}}+2 \frac{d \rho}{d \tilde{x}^{+}} \frac{d x^{+}}{d \tilde{x}^{+}}=0
$$

Thus,

$$
\frac{d}{d \tilde{x}^{+}}\left(\log \frac{d x^{+}}{d \tilde{x}^{+}}\right)=-2 \frac{d \rho}{d \tilde{x}^{+}},
$$

which is then solved to give

$$
\frac{d \tilde{x}^{+}}{d x^{+}}=C\left(x_{0}^{-}\right) e^{2 \rho\left(x^{+}, x_{0}^{-}\right)},
$$

where $C\left(x^{-}\right)$is a constant of integration which we will soon determine. Similarly, at fixed $x^{+}=x_{0}^{+}$,

$$
\frac{d \tilde{x}^{-}}{d x^{-}}=C\left(x_{0}^{+}\right) e^{2 \rho\left(x_{0}^{+}, x^{-}\right)} .
$$

Thus we make calculations of $\tilde{T}$, as defined by

$$
\tilde{T}_{ \pm \pm} \equiv\left(\frac{d \tilde{x}^{ \pm}}{d x^{ \pm}}\right)^{2} T_{ \pm \pm}=\frac{C\left(x_{0}^{\mp}\right)}{e^{4 \rho\left(x^{ \pm}\right)}} T_{ \pm \pm} .
$$

This gives us an observable measure of the energy flux that is independent of the choice in coordinates.

We choose the normalization constants, $C\left(x_{0}^{-}\right), C\left(x_{0}^{+}\right)$in (55) and (56), so the affine coordinates coincide with asymptotically Minkowskian coordinates far from the black hole. This normalization will not be necessary in the RN case, because there the coordinates are already asymptotically Minkowskian. For the DW model, we choose $C\left(x_{0}^{-}\right)$, $C\left(x_{0}^{+}\right)$with the requirement that along some large chosen value of $x^{+}$, termed $x^{+}=x_{\infty}^{+}$, and along a similarly chosen $x^{-}=x_{\infty}^{-}$,

$$
d s^{2}=-d \tilde{x}^{+} d \tilde{x}^{-}
$$

so that,

$$
e^{2 \rho} \frac{d x^{+}}{d \tilde{x}^{+}} \frac{d x^{-}}{d \tilde{x}^{-}}=1 .
$$

Given that asymptotically $e^{-2 \rho}=M-x^{+} x^{-}$, then this amounts to requiring that at $x^{-}=x_{0}^{-}$

$$
\frac{d x^{+}}{d \tilde{x}^{+}}=e^{-2 \rho\left(x^{+}, x_{0}^{-}\right)} \frac{\sqrt{M-x_{\infty}^{+} x_{\infty}^{-}}}{M-x_{\infty}^{+} x_{0}^{-}}
$$

and at $x^{+}=x_{0}^{+}$

$$
\frac{d x^{-}}{d \tilde{x}^{-}}\left(x_{0}^{+}\right)=e^{-2 \rho\left(x_{0}^{+}, x^{-}\right)} \frac{\sqrt{M-x_{\infty}^{+} x_{\infty}^{-}}}{M-x_{0}^{+} x_{\infty}^{-}} .
$$

Using these we obtain meaningfully normalized null affine coordinates with which to calculate the stress-energy tensors of (57). 


\section{RESULTS}

Careful analysis limits the range of values of our variables, $(\gamma, \mu$ and $\kappa$, in the DW model, $Q$ and $\kappa$ in the RN) for which numerical errors are sufficiently small to permit unambiguous statements. We compared our results against the classical solutions to gauge the buildup of numerical error. These numerical constraints prevent us from making the back-reaction arbitrarily weak. A useful measure of the strength of the back-reaction is the ratio of the time-scale of the quantum evaporation to the light-crossing time of the black hole. These time-scales may be extracted by inspection of the equations of motion, with the results that $t_{\text {light }} / t_{\text {evap }}=\kappa / Q^{2}$ for the $\mathrm{RN}$ model, and $t_{\text {light }} / t_{\text {evap }}=\kappa / \mu$ for the DW model. We quote the results for the regime when this ratio is of order one, where numerical errors are negilible. The qualitative behavior of the solutions does not change as $\kappa$ is made smaller, as far as we have been able to determine. Of course, the semi-classical approximation to the evaporation is no less valid when we do not impose the weak back-reaction condition.

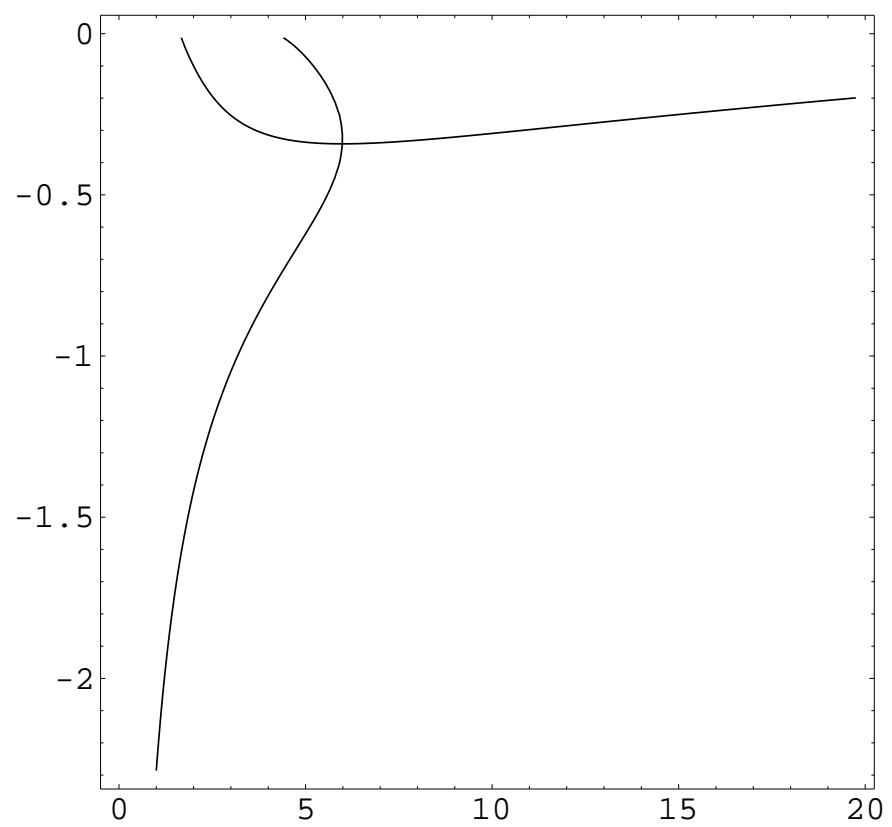

FIG. 1. DW model: The outer horizon is shown as it recedes to meet the inner horizon, in turn moving out, at $\phi_{0}$, the extremal radius. $x^{+}$and $x^{-}$are the $x$ and $y$ axes respectively. $[\gamma=8 ; \mu=15 ; \kappa=10 ; \Delta M=1.5]$

A typical contour plot is shown for each model in FIG. 1 and FIG. 2. As a shock mass is sent in, the two apparent horizons, given by the zeroes of $\partial_{+} \phi$, split apart, with the outer apparent horizon moving past $\phi_{0}$, while the inner horizon moves behind this radius. Immediately afterwards, the outer horizon begins to recede and meets the inner horizon back at the extremal radius $\phi_{0}$ (the value of $\phi$ at the position of the horizon of the initial extremal black hole).

It is useful to regard the separation of the horizons along the $x^{-}$direction as a measure of the excitation energy of the extremal black hole (this statement can be made more precise in the adiabatic approximation [15, 13]). This indicates the semi-classical solutions break down before the meeting point of the apparent horizons since here the energy of an emitted quanta inevitably becomes comparable to the energy of excitation above the extremal ground state [7]. Evolving for points in the causal future of this endpoint would no longer be consistent with the semi-classical approximation.

As discussed in [13], the Ricci scalar curvature calculated along a given contour of $\phi$ (see FIG. 3 and FIG. [) demonstrates a return to the extremal quantity as evaporation proceeds, strongly indicating a smooth evaporation process. This is to say that an observer at a fixed radius outside of the black hole sees the same physical environment towards the endpoint of evaporation as was seen before the shock mass was introduced.

For the DW model, we also evaluate $\tilde{T}_{ \pm \pm}$(as shown in FIG. 司 and FIG. 6), calculated from (57) along the fixed $\phi$ contour in FIG. 11. The behavior of these quantities is similarly consistent with a smooth evaporation process, returning the near-extremal black hole back to the extremal ground state. These fluxes are non-zero initially, but approach zero during evaporation. In the RN case, however, the flux perceived by an observer at a fixed radius outside of the black hole does not go to zero. This is evident from inspecting the behavior of $T_{--}$, shown in FIG. 8, whose absolute value does not decrease monotonically, indeed eventually rising again. This is a not an entirely unexpected 


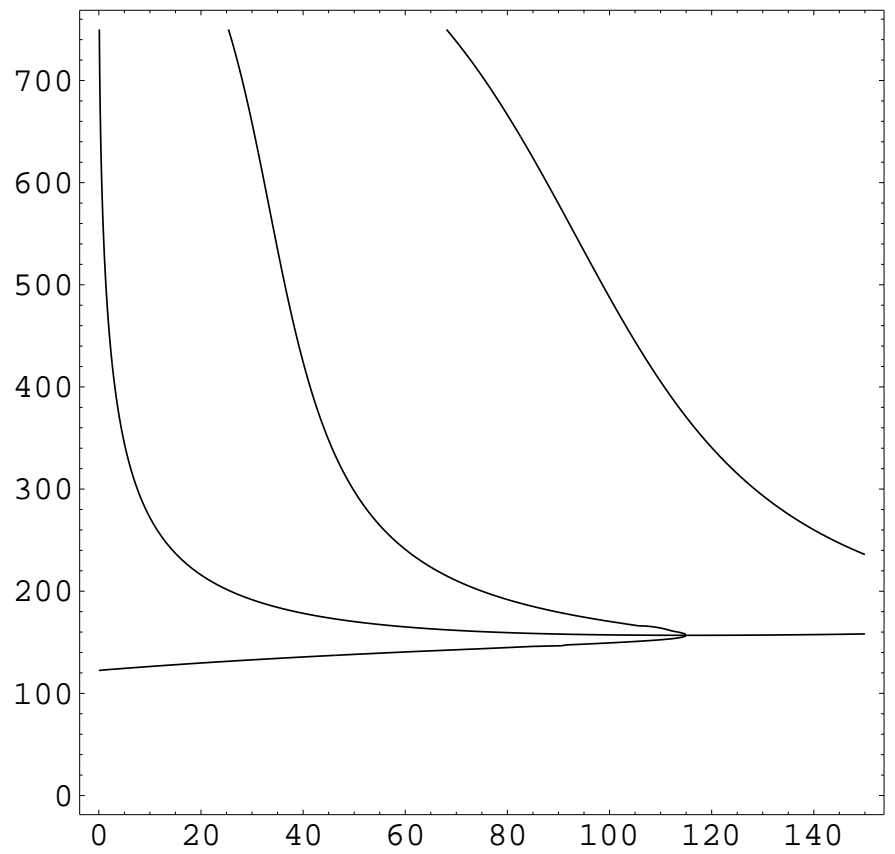

FIG. 2. RN model: The outer horizon is shown as it recedes to meet the inner horizon, in turn moving out, at $\tilde{\phi}_{0}$, the extremal radius. $x^{+}$and $x^{-}$are the $x$ and $y$ axes respectively. The additional contour lying above these is a remnant of $\tilde{\phi}_{0}$. No particular meaning has been attributed to this as it lies in a causally inaccessible region. $[Q=\sqrt{60} ; \kappa=40 ; \Delta M=.2]$

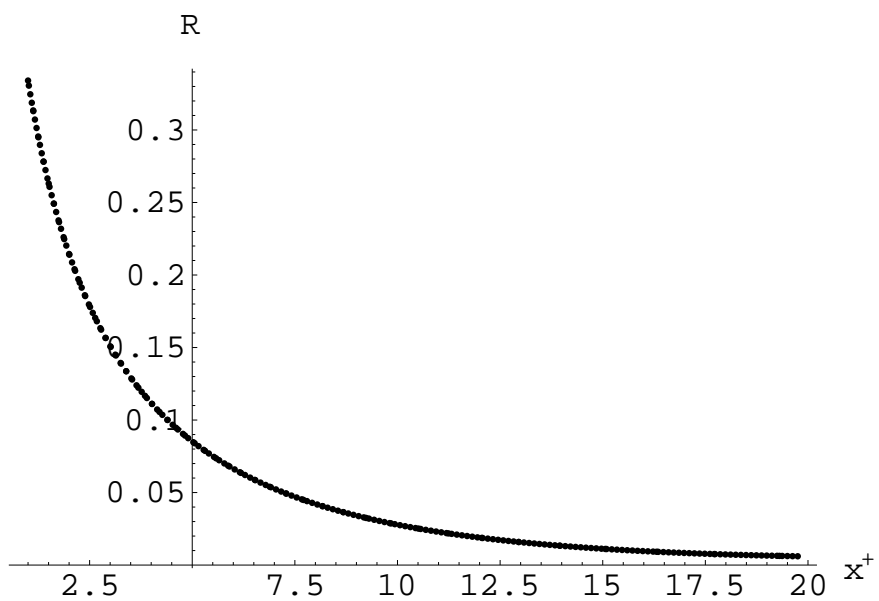

FIG. 3. DW model: The Ricci scalar curvature, $R$, calculated along the contour $\phi=-1.3$ throughout the evaporation process. The asymptotic value to which $R$ settles coincides with its extremal value. $[\gamma=8 ; \mu=15 ; \kappa=10 ; \Delta M=1.5]$

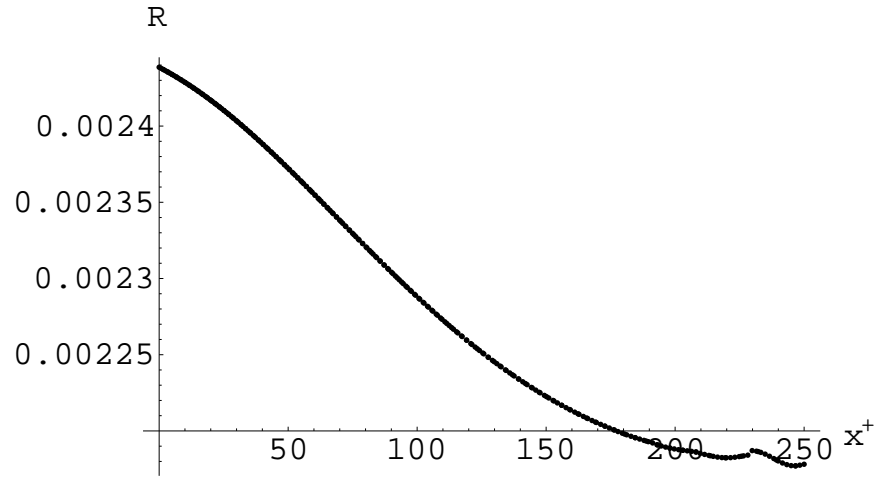

FIG. 4. RN model: The Ricci scalar curvature, $R$, calculated along the contour $\tilde{\phi}=15$ throughout the evaporation process. The asymptotic value to which $R$ settles coincides with its extremal value. $[Q=\sqrt{60} ; \kappa=40 ; \Delta M=.2]$ 


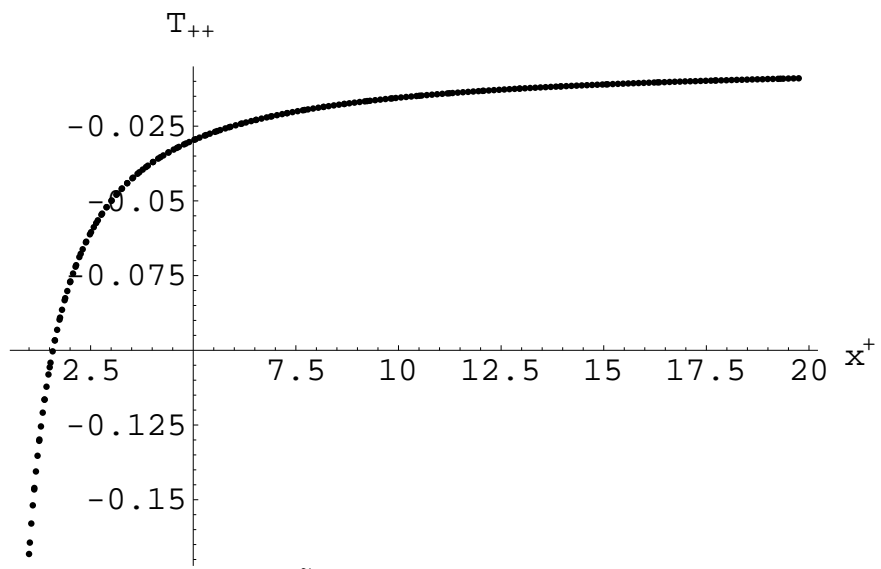

FIG. 5. DW model: $\tilde{T}_{++}$calculated along the contour $\phi=-1.3$ varies smoothly throughout the evaporation process. The data suggests that the flux $\tilde{T}_{++}$approaches zero, or a small constant, as the shock mass is evaporated away. $[\gamma=8 ; \mu=15 ; \kappa=10 ; \Delta M=1.5]$

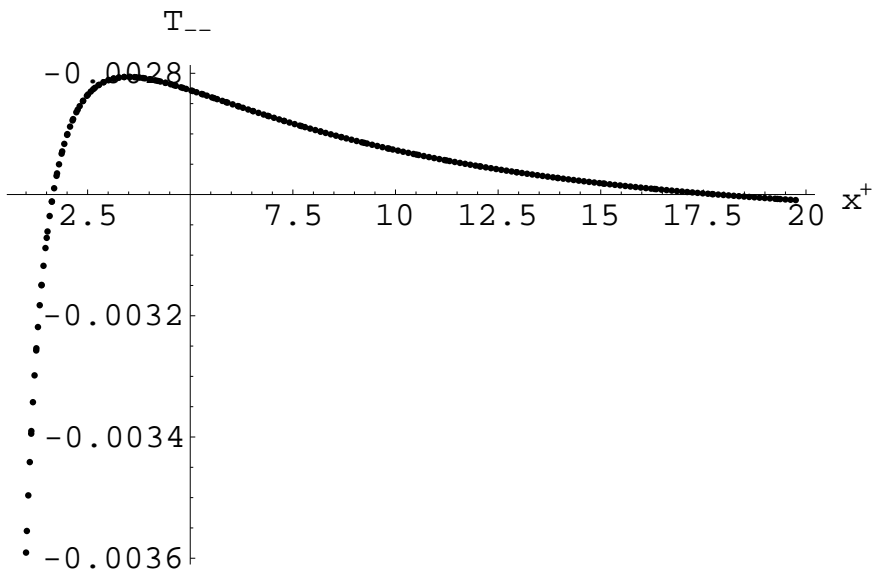

FIG. 6. DW model: $\tilde{T}_{--}$calculated along the contour $\phi=-1.3$ varies smoothly throughout the evaporation process. As the endpoint of evaporation is reached, the data suggests that this quantity approaches some constant value. $[\gamma=8 ; \mu=15 ; \kappa=10 ; \Delta M=1.5]$

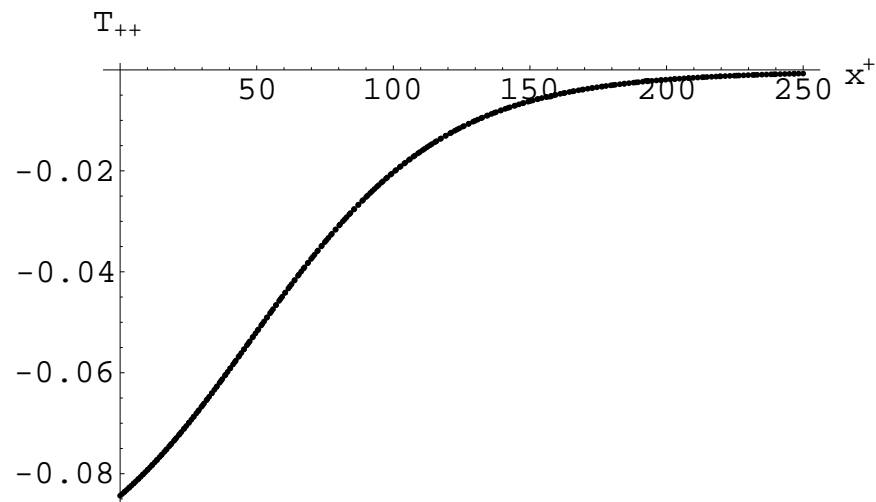

FIG. 7. RN model: $\tilde{T}_{++}$calculated along the contour $\tilde{\phi}=15$ throughout the evaporation process. After the shock mass is introduced at $x_{0}^{+}$, the flux $\tilde{T}_{++}$, which is negative, effectively approaches zero. $[Q=\sqrt{60} ; \kappa=40 ; M=5.641 ; \Delta M=.2]$ 


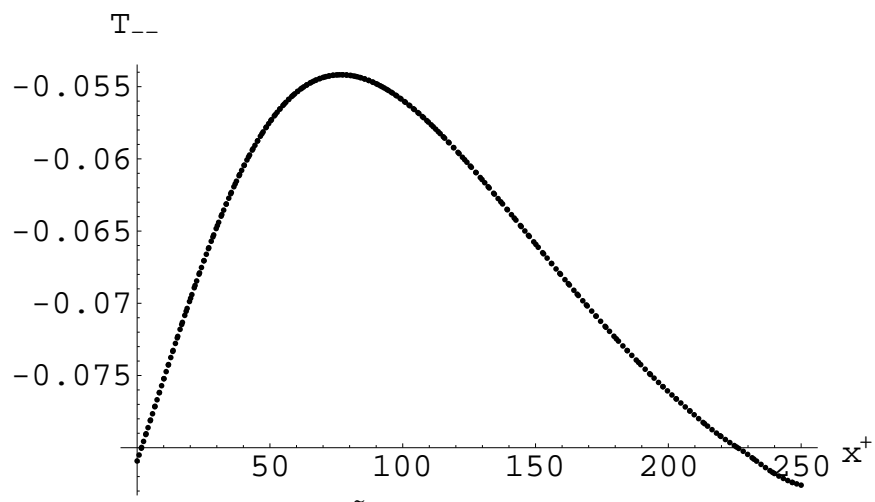

FIG. 8. RN model: $\tilde{T}_{--}$calculated along the contour $\tilde{\phi}=15$ throughout the evaporation process. $[Q=\sqrt{60} ; \kappa=40 ; \Delta M=.2]$

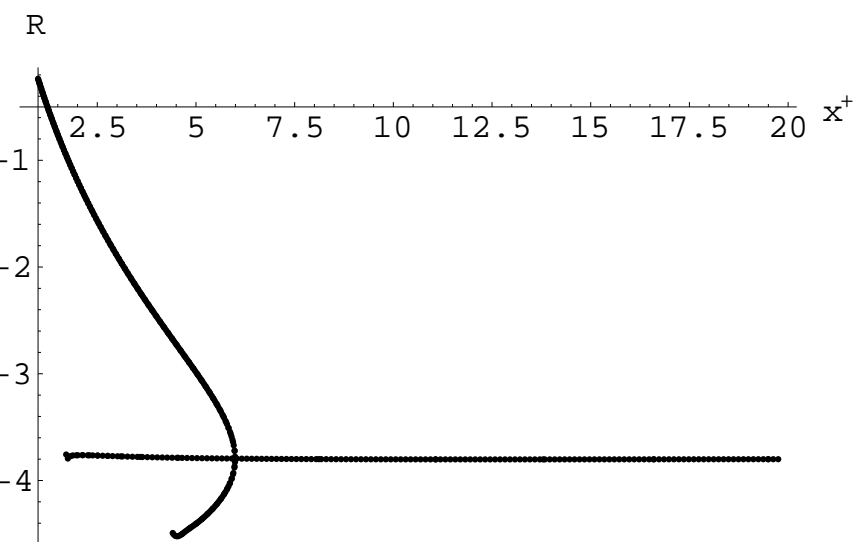

FIG. 9. DW model: The Ricci scalar curvature, $R$, evaluated at points along the outer horizon, the inner horizon, and $\phi_{0}$, overlapped. The absolute value of the curvature at the outer horizon is increasing, while decreasing at the inner horizon. When the horizons meet again at the extremal radius, $\phi_{0}$, the curvature is at its extremal value, indicating that the horizon environment has returned to its original state. $[\gamma=8 ; \mu=15 ; \kappa=10 ; \Delta M=1.5]$

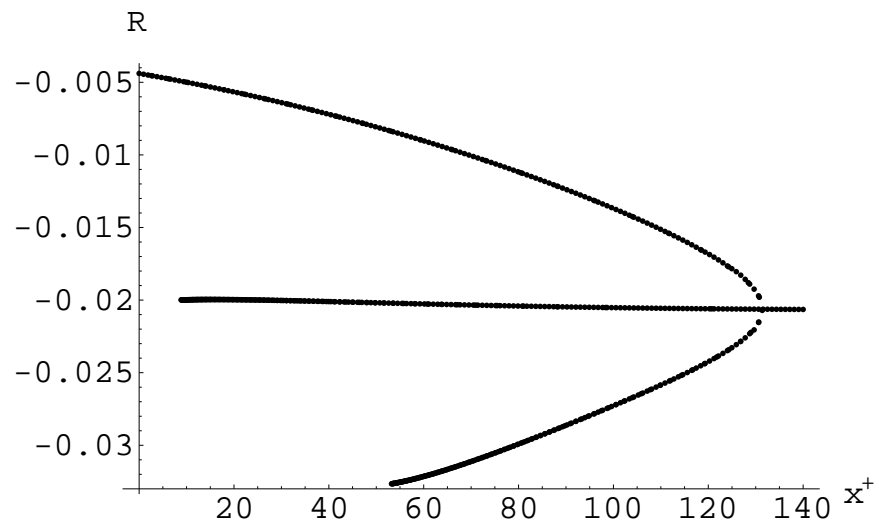

FIG. 10. RN model: The Ricci scalar curvature, $R$, evaluated at points along the outer horizon, the inner horizon, and $\tilde{\phi}_{0}$, overlapped. The absolute value of the curvature at the outer horizon is increasing, while decreasing at the inner horizon. When the horizons meet again at the extremal radius, $\tilde{\phi}_{0}$, the curvature is at its extremal value, indicating that the horizon environment has returned to its original state. $[Q=\sqrt{60} ; \kappa=40 ; \Delta M=.2]$ 
result, since the affine coordinates are not true asymptotically Minkowskian coordinates, so the flux need not vanish at infinity. $T_{++}$(FIG. 7), however, does approach a zero value with increasing $x^{+}$.

In order to directly test for instabilities in the inner or outer apparent horizon we also inspect the behavior of the stress-energy momentum and the Ricci scalar curvature for the contours of $\partial_{+} \phi=0$ along the outer and inner apparent horizons in order to see if an instability arises. FIG. 9 and FIG. 10 appear to indicate that the scalar curvature evolves smoothly throughout evaporation and returns to its extremal value. Meanwhile, FIG's. 11 - 18 demonstrate that for both models the absolute flux through the horizons decreases during the evaporation, consistent with a decreasing black hole mass. More importantly, there is no indication of a buildup of energy behind either the inner or outer apparent horizon. The smooth variation of $T_{++}$and $T_{--}$in these regions suggests a return, through evaporation, to the extremal ground state after the initial excitation.

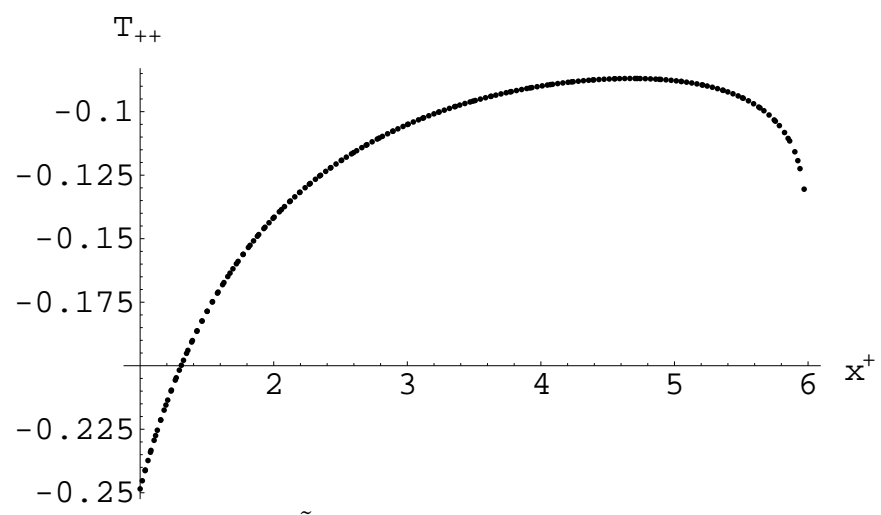

FIG. 11. DW model: $\tilde{T}_{++}$evaluated at points along the outer horizon, shown as it varies smoothly for increasing $x^{+}$. $[\gamma=8 ; \mu=15 ; \kappa=10 ; \Delta M=1.5]$

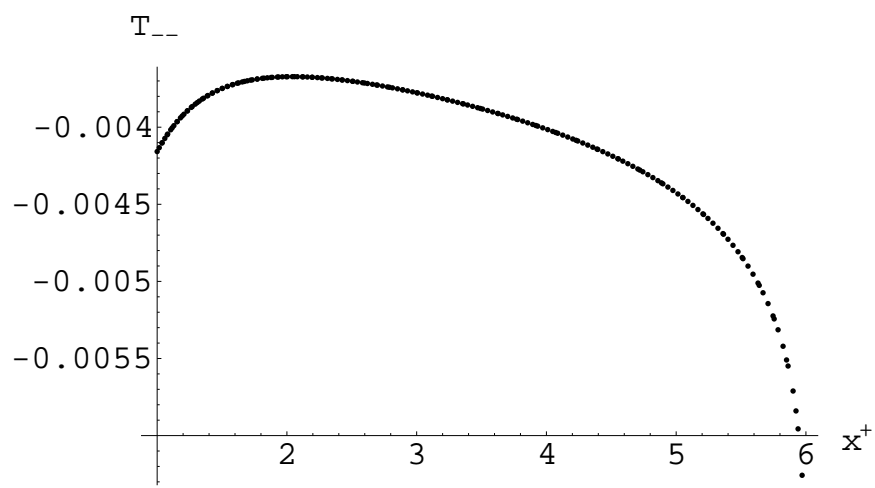

FIG. 12. DW model: $\tilde{T}_{--}$evaluated at points along the outer horizon, shown as it varies smoothly for increasing $x^{+}$. $[\gamma=8 ; \mu=15 ; \kappa=10 ; \Delta M=1.5]$

\section{DISCUSSION}

The pictures emerging from both the DW and RN case were consistent. After the injection of shock matter, the near-extremal black hole evaporates and returns to the extremal ground state. The outer and inner horizon experience no distressing energy instabilities and come back together, rejoining at the extremal radius. Furthermore, studies of the Ricci scalar and the stress-energy tensors calculated along null affine geodesics, indicate that for observers outside the black hole, the environment returns to its original state.

The original motivation for studying the evaporation of charged black holes was to shed light on the black hole information puzzle. Quantum mechanics necessarily requires unitarity. However, semi-classically, the emitted radiation from black holes possesses a thermal distribution. This leads to the information puzzle: what happens to the information contained in the ingoing matter once the mass of the black hole is radiated away?

While it may be that physics in the real universe is simply not unitary 23,24, other resolutions have nevertheless been suggested. One such potential resolution of the paradox is that the information of the ingoing matter becomes 


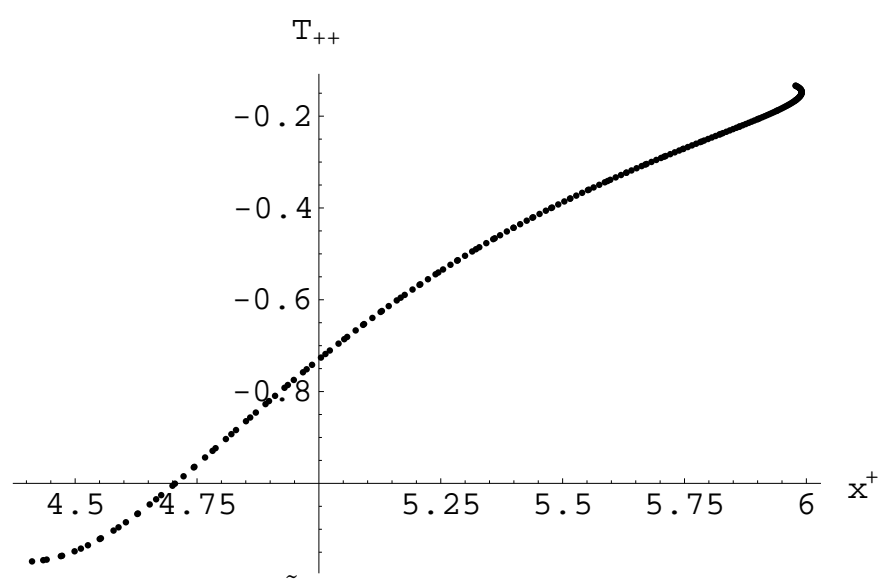

FIG. 13. DW model: $\tilde{T}_{++}$evaluated at points along the inner horizon, shown as its value decreases smoothly for increasing $x^{+} .[\gamma=8 ; \mu=15 ; \kappa=10 ; \Delta M=1.5]$

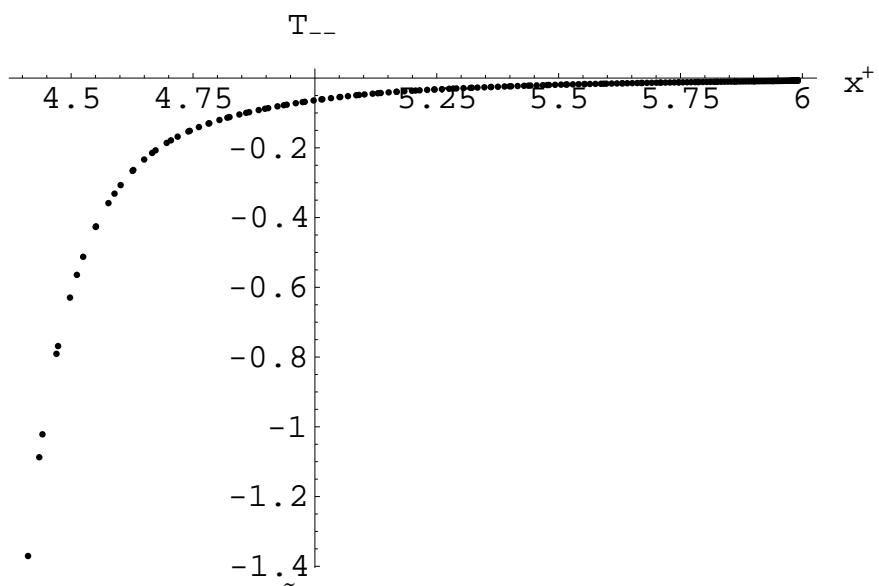

FIG. 14. DW model: $\tilde{T}_{--}$evaluated at points along the inner horizon, shown as it varies for increasing $x^{+}$. This flux goes to zero as the shock mass evaporates away. $[\gamma=8 ; \mu=15 ; \kappa=10 ; \Delta M=1.5]$

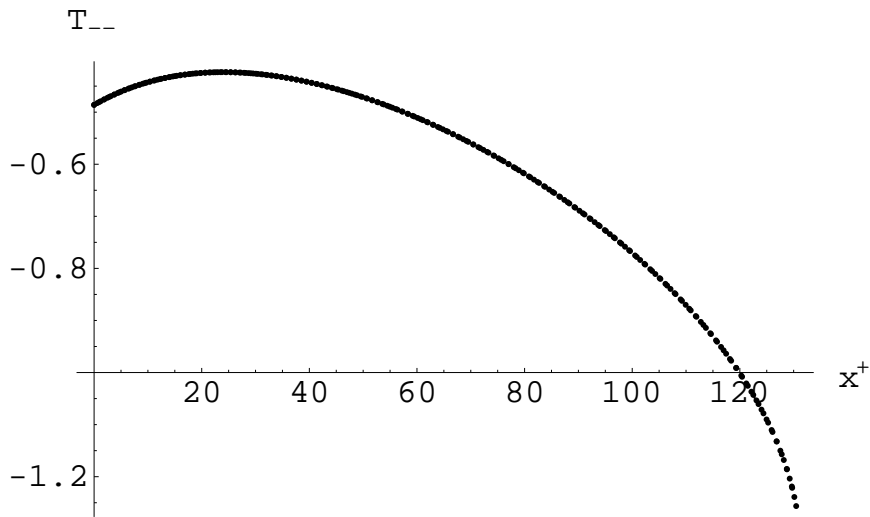

FIG. 15. RN model: $\tilde{T}_{--}$evaluated at points along the outer horizon, shown as it varies smoothly for increasing $x^{+}$. $[Q=\sqrt{60} ; \kappa=40 ; \Delta M=.2]$ 


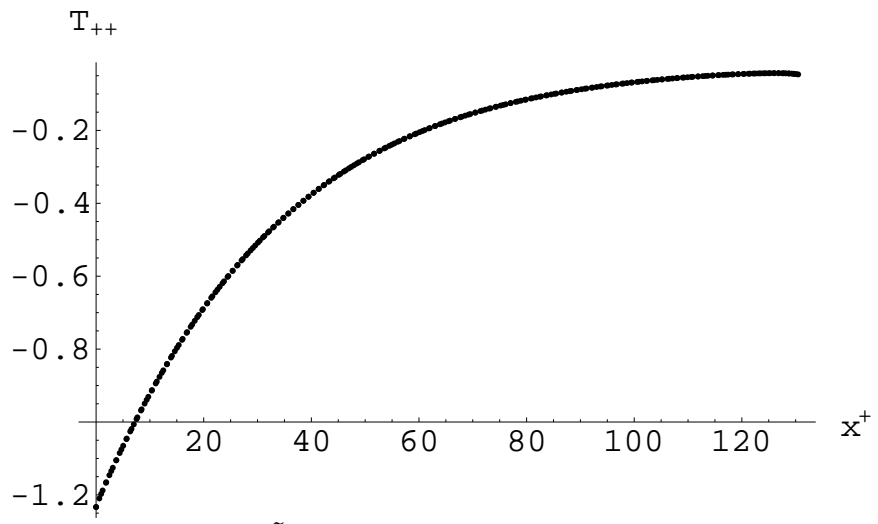

FIG. 16. RN model: $\tilde{T}_{++}$evaluated at points along the outer horizon, shown as it varies smoothly for increasing $x^{+}$. The $\tilde{T}_{++}$flux through the outer horizon appears to vanish as evaporation proceeds. $[Q=\sqrt{60} ; \kappa=40 ; \Delta M=.2]$

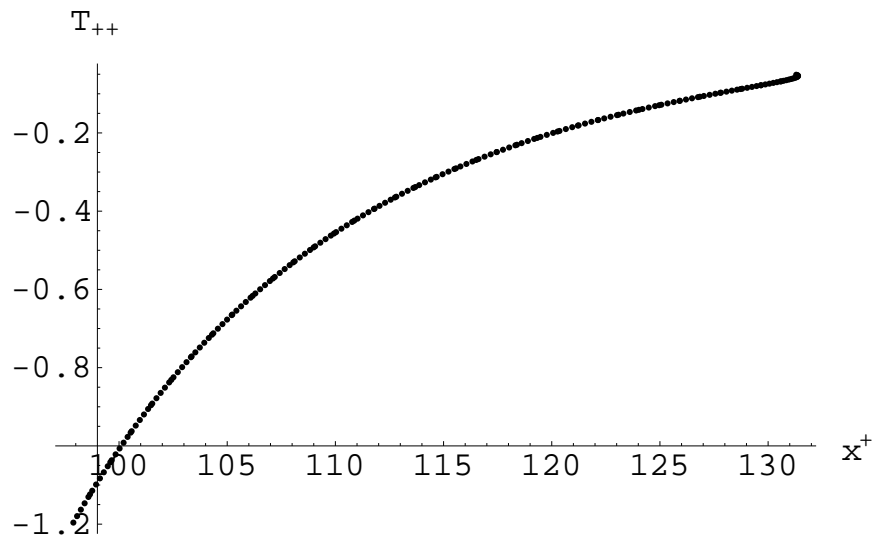

FIG. 17. RN model: $\tilde{T}_{++}$evaluated at points along the inner horizon, shown as it varies for increasing $x^{+}$. This flux decreases in absolute value throughout the evaporation process. $[Q=\sqrt{60} ; \kappa=40 ; \Delta M=.2]$

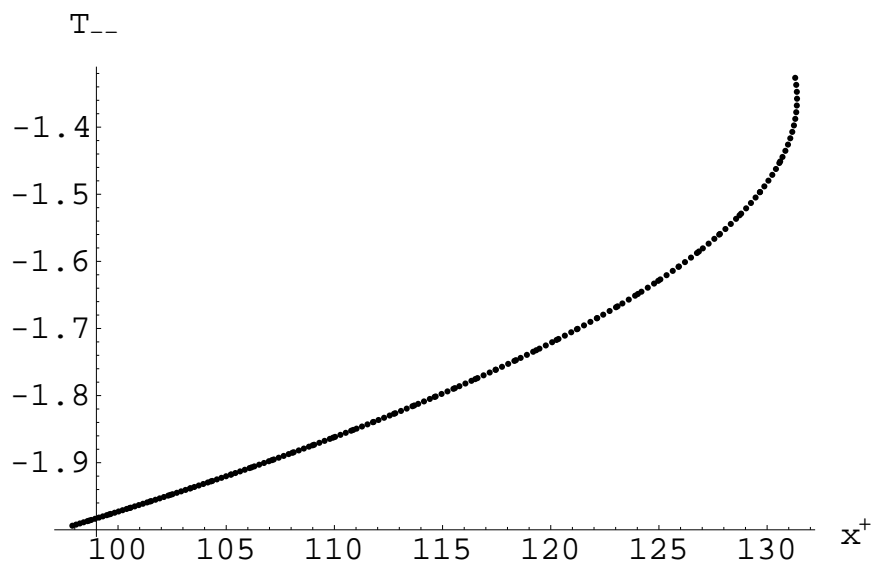

FIG. 18. RN model: $\tilde{T}_{--}$evaluated at points along the inner horizon, shown as it varies smoothly for increasing $x^{+}$. $[Q=\sqrt{60} ; \kappa=40 ; \Delta M=.2]$ 
stored in stable remnants that remain at the end of evaporation. These remnants would apparently need to contain an arbitrarily large amount of information despite possessing a Planck-sized mass [26]. In this regard, extremal black holes hold some promise; behind the charged black hole horizon lies an infinite throat region [3] that can apparently store an arbitrary amount of information. This fact is also manifested by the infinite degeneracy of the extremal ground state [15] at the semi-classical level. Thus extremal black holes represent the testing ground for the remnant resolution to the information paradox [1,2]. There is some debate as to whether or not the pair production of such charged black holes occurs at a finite rate [3,1,27,2], given the infinite degeneracy of the ground state. However, this infinite degeneracy is likely a result of the semiclassical averaging over the quantum geometries. This should not occur in a full quantum treatment. For example, D-brane calculations demonstrate that summing over microstates in fact yields a finite Bekenstein-Hawking entropy [28]. Matrix theory also indicates a similar result [29]. This may lay to rest infinite production concerns. Our studies did not reveal an instability in the evolution of excited extremal black holes. Thus as it stands, we cannot yet rule them out as the ultimate devices for information storage.

Another suggested resolution to the information puzzle proposes that higher order quantum corrections would reveal that the outgoing radiation is not strictly thermal, carrying off information about the ingoing radiation in the form of quantum correlations with the ingoing matter. This, however, while at first seeming to be a most agreeable resolution, presents other theoretical difficulties. Once matter disappears behind the horizon, it becomes causally dissociated from the outside region. Therefore such cross-horizon correlations would necessarily violate causality, unless some sort of information stripping were to occur at the horizon itself. At the semi-classical level, however, we do not expect anything special to occur at the horizon in the frame of reference of the in-falling observer. This issue can be resolved by appealing to a "stretched horizon" membrane picture [30], based on the principle of black hole complementarity. This principle asserts that matter which has fallen past the event horizon and the Hawking radiation emitted from there are not different objects; they are equivalent and complementary descriptions from reference frames which can be related to each other by large Lorentz boosts. Since there is no communication to outside of the black hole once an observer has passed in, there is no logical contradiction by prescribing that unitarity remains valid entirely outside of the horizon. Furthermore, the contribution of non-local terms arising from string theory [31,32] may account for cross-horizon correlations which will nevertheless be undetectable by any single observer. That is, if we do not require a local field theory, except at low energy, it becomes possible to construct a fully self-consistent causal theory where field operators behind and in front of the horizon will not commute [33]. String theory and the existence of quantum correlations between the ingoing and outgoing matter then ultimately promises to contain the key for resolving the information puzzle.

The models we have considered here suggest that at the semi-classical level, upon entering the black hole, information is lost to the outside world. However, while it becomes inaccessible, the information enters the infinite asymptotic throat region behind the horizon and is not actually destroyed. While this picture permits remnants to contain the "lost" information, it still remains possible that the non-local string theory terms do indeed represent the real universe and the information does return to the outside region. Our success lies in showing that the semi-classical treatment of charged black hole evaporation does not breakdown sooner than would be expected from [7], despite heuristic arguments of Jacobson [6] to the contrary. Results presented here are also consistent with analytical results found in [8].

[1] S. B. Giddings, "Why aren't black holes infinitely produced?," Phys. Rev. D 51, 6860 (1995) [hep-th/9412159].

[2] S. B. Giddings, "The Black hole information paradox," hep-th/9508151.

[3] T. Banks, M. O'Loughlin and A. Strominger, "Black hole remnants and the information puzzle," Phys. Rev. D 47, 4476 (1993) hep-th/9211030.

[4] C. G. Callan, S. B. Giddings, J. A. Harvey and A. Strominger, "Evanescent black holes," Phys. Rev. D 45, 1005 (1992) hep-th/9111056.

[5] D. A. Lowe, "Semiclassical approach to black hole evaporation," Phys. Rev. D 47, 2446 (1993) hep-th/9209008.

[6] T. Jacobson, "Semiclassical decay of near-extremal black holes," Phys. Rev. D 57, 4890 (1998) hep-th/9705017.

[7] J. Preskill, P. Schwarz, A. Shapere, S. Trivedi and F. Wilczek, "Limitations on the statistical description of black holes," Mod. Phys. Lett. A 6, 2353 (1991).

[8] K. Diba and D. A. Lowe, "The stress-energy tensor in soluble models of spherically symmetric charged black hole evaporation," hep-th/0107137.

[9] A. Fabbri, D. J. Navarro and J. Navarro-Salas, "Evaporation of near-extremal Reissner-Nordström black holes," [hepth/0004027v1]. 
[10] A. Fabbri, D. J. Navarro and J. Navarro-Salas, "Evaporation of near-extremal Reissner-Nordström black holes," Phys. Rev. Lett. 85, 2434 (2000) hep-th/0004027.

[11] A. Fabbri, D. J. Navarro and J. Navarro-Salas, "Quantum evolution of near-extremal Reissner-Nordstroem black holes," Nucl. Phys. B 595, 381 (2001) arXiv:hep-th/0006035.

[12] A. Fabbri, D. J. Navarro and J. Navarro-Salas, "Low-energy scattering of extremal black holes by neutral matter," arXiv:hep-th/0110294.

[13] D. A. Lowe and M. O'Loughlin, "Nonsingular black hole evaporation and 'stable' remnants," Phys. Rev. D 48, 3735 (1993) hep-th/9305125.

[14] S. P. Trivedi, "Semiclassical extremal black holes," Phys. Rev. D 47, 4233 (1993) hep-th/9211011].

[15] A. Strominger and S. P. Trivedi, "Information consumption by Reissner-Nordström black holes," Phys. Rev. D 48, 5778 (1993) hep-th/9302080.

[16] S. B. Giddings and A. Strominger, "Dynamics of extremal black holes," Phys. Rev. D 46, 627 (1992) hep-th/9202004.

17] C. Barbachoux and A. Fabbri, "Semiclassical zero temperature black holes in spherically reduced theories," [hepth/0201133.

[18] D. A. Lowe, Phys. Rev. Lett. 87, 029001 (2001) gr-qc/0011053.

[19] A. M. Polyakov, "Quantum Geometry Of Bosonic Strings," Phys. Lett. B 103, 207 (1981).

[20] A.M. Polyakov, Gauge Fields and Strings, Harwood Academic Publishers, 1987.

[21] T. Banks and M. O'Loughlin, "Nonsingular Lagrangians for two-dimensional black holes," Phys. Rev. D 48, 698 (1993) hep-th/9212136.

[22] S. W. Hawking and J. M. Stewart, "Naked and thunderbolt singularities in black hole evaporation," Nucl. Phys. B 400, 393 (1993) hep-th/9207105.

[23] S. W. Hawking, "Particle Creation By Black Holes," Commun. Math. Phys. 43, 199 (1975).

[24] S. W. Hawking, "Breakdown Of Predictability In Gravitational Collapse," Phys. Rev. D 14, 2460 (1976).

[25] B. Birnir, S. B. Giddings, J. A. Harvey and A. Strominger, "Quantum black holes," Phys. Rev. D 46, 638 (1992) [hepth/9203042.

[26] J. Preskill, "Do black holes destroy information?," hep-th/9209058.

[27] L. Susskind, "Trouble for remnants," hep-th/9501106.

[28] A. Strominger and C. Vafa, "Microscopic Origin of the Bekenstein-Hawking Entropy," Phys. Lett. B 379, 99 (1996) hep-th/9601029.

[29] D. A. Lowe, "Statistical origin of black hole entropy," Phys. Rev. Lett. 81, 256 (1998) hep-th/9802173; D. Kabat, G. Lifschytz and D. A. Lowe, "Black hole thermodynamics from calculations in strongly coupled gauge theory," Phys. Rev. Lett. 86, 1426 (2001) [Int. J. Mod. Phys. A 16, 856 (2001)] arXiv:hep-th/0007051]; D. Kabat, G. Lifschytz and D. A. Lowe, "Black hole entropy from non-perturbative gauge theory," Phys. Rev. D 64, 124015 (2001) arXiv:hep-th/0105171.

[30] L. Susskind, L. Thorlacius and J. Uglum, "The Stretched horizon and black hole complementarity," Phys. Rev. D 48, 3743 (1993) hep-th/9306069.

[31] D. A. Lowe, "The Planckian conspiracy: String theory and the black hole information paradox," Nucl. Phys. B 456, 257 (1995) hep-th/9505074.

[32] D. A. Lowe, J. Polchinski, L. Susskind, L. Thorlacius and J. Uglum, "Black hole complementarity versus locality," Phys. Rev. D 52, 6997 (1995) hep-th/9506138.

[33] D. A. Lowe, L. Susskind and J. Uglum, "Information spreading in interacting string field theory," Phys. Lett. B 327, 226 (1994) hep-th/9402136. 